\title{
Mapping the Structure of an Organic Monolayer
}

\author{
Shaul Aloni ${ }^{1}$, Virginia Altoe ${ }^{1}$, Allard Katan ${ }^{2}$, Florent Martin $^{2}$ and Miquel Salmeron ${ }^{1,2}$ \\ ${ }^{1}$ The Molecular Foundry and ${ }^{2}$ Materials Science Division, Lawrence Berkeley National Laboratory, \\ Berkeley, CA 94706
}

Monolayers and ultrathin films of organic materials are studied intensively because of their practical applications in many disciplines, including nanofabrication, biosensing and photovoltaics. In such films, variations of crystalline order over nanometer length scales are key to functionality. Until now, high-resolution structural information on monolayers was only available by x-ray scattering studies that sample many microns in size or scanning probe techniques that provide lattice periodicity in a small area, but are not feasible for large-area mapping of structure. Our technique provides fast and unambiguous means of obtaining local structure variation of large sample areas with a resolution of $100 \mathrm{~nm}$, which may be improved tenfold using readily available technology. The organic semiconductor Pentathiophene Butyric Acid (5TBA) was synthesized, dissolved in chloroform and deposited onto a Langmuir-Blodgett trough. Molecules self-assembled at the airwater interface then transferred onto $20 \mathrm{~nm}$ thin ultra-flat SiN membranes and analyzed by TEM. Since small variations in sample preparation alter the molecular film thickness, samples were always screened by AFM to confirm the presence of 5TBA monolayers (fig. 1b). As with many organic materials, the electron beam damages the 5TBA monolayers. All structural information has to be obtained with a total dose of $<50 \mathrm{e}^{-} / \AA^{2}$, rendering standard high-resolution electron microscopy methods infeasible.

The morphology of the monolayer consists of contiguous islands $0.5-2 \mu \mathrm{m}$ in size, and porous regions. The spatially resolved diffraction patterns show that the islands are made up of one or two well-ordered crystalline domains, which differ only by their lattice orientation. The lack of the $(0,1)$ and $(1,0)$ reflections (fig. 1a) indicates that their structure is described in 2 dimensions by the p2gg space group, and is consistent with a herringbone arrangement of two molecules per unit cell (fig 1c). The lattice parameters are $a=5.35 \pm 0.12 \AA$ and $b=7.46 \pm 0.15 \AA$. A simulated diffraction pattern obtained from this model (fig 1d) shows excellent agreement with the experimental results. Neither the space group, nor the presence of multiple domains within an island could previously be obtained with other techniques.

The orientation of the $(0,1)$ direction of these domains is depicted in fig. 1e by yellow arrows. The boundaries between domains are sharp, with many examples of complete transition from one direction to the other within a single $100 \mathrm{~nm}$ pixel. Diffraction patterns from porous regions of the film consist of a large number of distinct spots. The spacing is the same as observed in the larger domains, suggesting that both regions have the same molecular structure but the porous phase lacks long-range order. This sub-monolayer morphology is characteristic of a 2D nucleation, growth and 
coalescence during solvent evaporation. The porous phase results from local solution heterogeneity, such as lack of nucleation centers.

In summary, we have demonstrated a simple and versatile method for high-resolution local structure determination of nanoscale domains of a one molecule thin organic material. This technique presents a major step forward in the characterization of 2D organic materials. It is not limited to our particular monolayer/substrate combination, and we expect our method to have widespread applications in materials research. For example, it could be used to study the degree of crystallinity and grain orientation in polymer thin films, or the structure of the interface between phases in nanoparticle/organic blends.
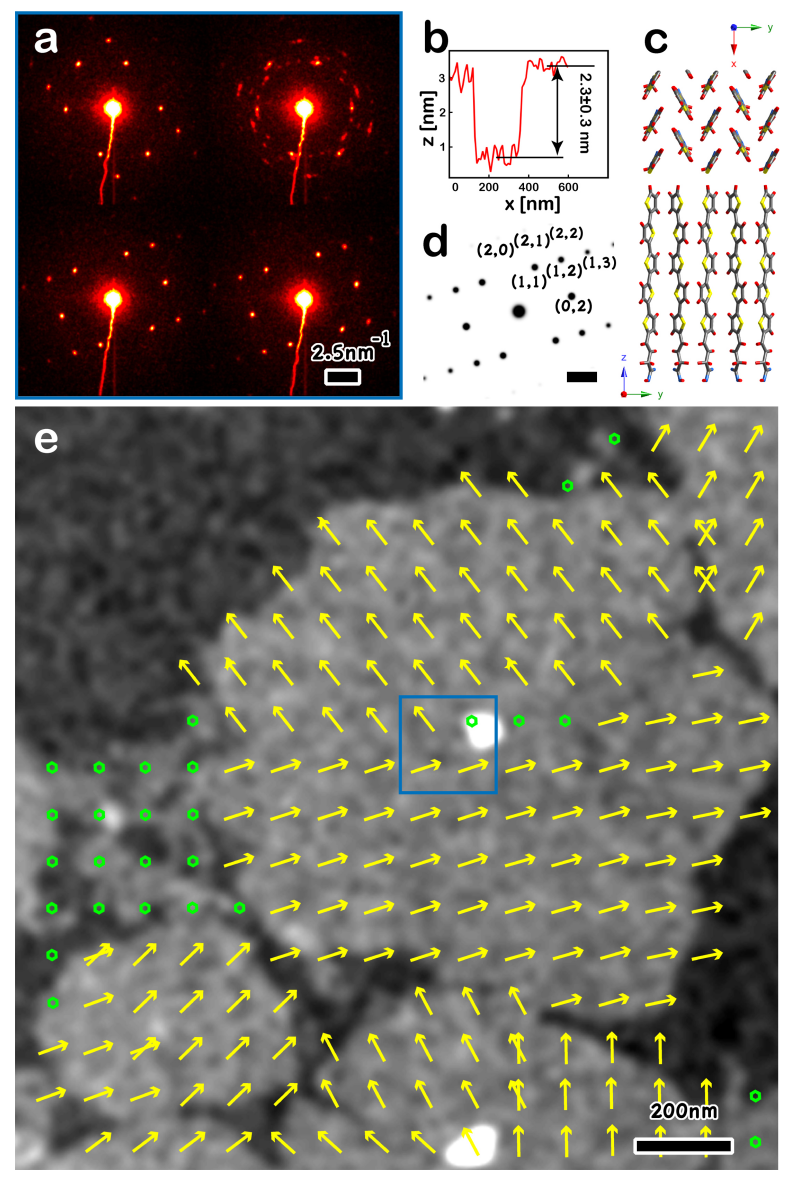

Figure 1. a) Diffraction patterns from 4 adjacent areas depicted by the blue rectangle in fig.1e. b) AFM cross-section of two 5TBA islands on a TEM grid, c) top view (upper) and side view (lower) of the proposed crystalline structure of the molecular film, d) simulated kinematic diffraction pattern, e) high resolution HAADF of the 5TBA monolayer. Yellow arrows depict the orientation of the $(0,1)$ crystallographic direction of ordered domains. Green hexagons mark locations with complex diffraction patterns. 